\title{
Fluidez verbal fonológica, morfológica y semántica en sujetos con enfermedad de Parkinson
}

\author{
Phonologic, morphologic and semantic verbal fluency in Parkinson disease subjects
}

\author{
María Francisca Alonso-Sánchez ${ }^{1}$ y Pedro Alfaro-Faccio ${ }^{2}$
}

${ }^{1}$ Fonoaudióloga. Doctora en Microbiología, Salud Pública y Medicina Preventiva. Investigadora en la Escuela de Fonoaudiología, Facultad de Salud, Universidad Santo Tomás, Viña del Mar,

Chile. E-mail: mariaalonsosa@santotomas.cl

${ }^{2}$ Doctor en Lingüística. Investigador en el Instituto de Literatura y Ciencias del Lenguaje, Pontificia Universidad Católica de Valparaíso, Chile. E-mail: pedro.alfaro@pucv.cl

Universidad Santo Tomás, Viña del Mar, Chile.

\section{Resumen}

La Enfermedad de Parkinson (EP) es una patología progresiva con degeneración del sistema dopaminérgico nigro-estriatal con disfunción de proyecciones frontales que genera alteraciones en funciones ejecutivas. Esta condición debería afectar particularmente la capacidad de evocar unidades léxicas y recuperarlas desde la memoria de largo plazo; no obstante, si bien se cuenta con datos acerca de este comportamiento, aún es necesario determinar qué subcomponentes del lenguaje se ven afectados a fin de comprender con mayor especificidad tanto la patología como su manifestación lingüística. En este marco, el objetivo del presente estudio consistió en describir el rendimiento en tareas de fluidez verbal de tipo fonológica, morfosintáctica, semántica y sus combinaciones en participantes con enfermedad de Parkinson (EP). Para ello, se realizó un estudio transversal con 42 sujetos, agrupados en Adulto Mayor Sano (AMS; $n=23$ ) y Adulto Mayor Diagnosticado con EP (EP; $n$ $=19$ ). Cada sujeto realizó un total de 15 tareas de fluidez verbal en las que debió evocar la mayor cantidad de unidades léxicas en 60 segundos, las que fueron, además, medidas en intervalos de 15 segundos. Los resultados permitieron observar diferencias estadísticamente significativas en las subtareas de tipo fonológica de fonema excluido, categoría gramatical, relaciones semánticas y de combinación campo léxico y fonema inicial. Estos datos parecen indicar que los sujetos con EP muestran un rendimiento significativamente inferior en tareas que exigen alto control inhibitorio, dado que las subtareas que combinan niveles de la lengua implican evocación e inhibición.

Palabras clave: Enfermedad de Parkinson; Fluidez fonológica, morfológica y semántica.

\begin{abstract}
Parkinson's disease (PD) is a progressive pathology that implies a degeneration of the nigro-striatal dopaminergic system with dysfunction of frontal projections, which generates alterations in executive functions, specifically in sustained attention, working memory, planning and cognitive flexibility, among others. In fact, this pathology has reper-
\end{abstract}


cussions on several language components. Particularly, there is evidence indicating that the ability to evoke lexical units and recover them from long-term memory or lexicon is affected. Although these types of tasks seem to be sensitive for the detection of Parkinson's disease, both in patients with mild cognitive impairment and another with dementia, it is still necessary to have evidence to determine which specific subcomponents of the language are affected, since this will allow us to understand with more specificity both the pathology and its linguistic manifestation, at the same time as to design therapies by support teams for this type of patients. In this context, verbal fluency (VF) tests could contribute to obtaining this type of information. VF is defined as an executive function that involves the access and evocation of clusters of linguistic information, and that is related to attention and working memory processes, since it is initiated by the activation of information groups through specific search strategies Moreover, it involves various linguistic functions, such as the naming, and executive functions of planning, working memory and initiation of behavior. Specifically, this type of task consists of requesting the subject under evaluation to nominate the largest number of elements of a lexical category according to a specific rule for a specific time, such as names of countries or word containing the phoneme / f / From there, it has been observed which associated language areas are activated: ventral-anterior lower frontal gyrus in categorical tasks, dorsal-posterior lower frontal gyrus in phonological tasks and parietal areas of the right hemisphere that are associated with executive functions and attentional processes in tasks of greater complexity. Within this framework, the aim of the present study was to describe the performance in tasks of verbal fluency of a phonological, morph syntactic, semantic type and their combinations in participants diagnosed with Parkinson's disease (PD). For this, a cross-sectional study was conducted with 42 subjects, grouped as healthy older adults
$($ AMS $; n=23)$ and older adults diagnosed with PD without dementia (EP; $n=19)$. The subjects of the control group had a mean age of 66 years $(\mathrm{SD}=6.9)$ and 11.5 years of schooling $(\mathrm{SD}=3.1)$; on the other hand, subjects with $\mathrm{PD}$ had a mean age of 71 years $(\mathrm{SD}=8.03)$ and 13.2 years of schooling $(\mathrm{SD}=3.9)$. Each subject performed a total of 15 verbal fluency tasks, in which he had to evoke the greatest number of lexical units in 60 seconds, which were also measured in intervals of 15 seconds. The results shown statistically significant differences in the sub-tasks of the phonemic type of excluded phoneme (FVFLE1: $p=$ .012 and FVFLE2: $p=.047)$, morph syntactic adjective categories (FVM2: $p=.005)$, in the synonymy relationships (FVS3: $p=.028)$ and lexical field and phonology (FVSF: $p=.004$ ). These data seem to indicate that subjects with Parkinson's disease show significantly lower performance in tasks that require high inhibitory control, since subtasks that combine tongue levels involve evocation and inhibition at the same time. These results are consistent with the pathophysiology of the condition.

Keywords: Parkinson Disease; Phonologic, morphologic and semantic verbal fluency

\section{Introducción}

La Enfermedad de Parkinson (EP) es una patología degenerativa, altamente prevalente, caracterizada por temblor, rigidez, bradicinesia e inestabilidad postural (Calne, Snow y Lee, 1992). Es una patología progresiva con degeneración del sistema dopaminérgico nigro-estriatal (Dubois y Pillon, 1996), con depósitos de cuerpos de Lewy y neuritas distróficas (Lippa et al., 2007), que afecta principalmente los ganglios basales y consecuentemente la vía talámica ventral anterior y medial dorsal con proyecciones al lóbulo frontal (Taylor, Saint-Cyr y Lang, 1986). Con respecto a las alteraciones cognitivas, y en consecuencia a la disfunción en proyecciones frontales, se han observado alteraciones en 
funciones ejecutivas (Perfetti et al., 2010; Petrova et al., 2010), específicamente en atención sostenida (Alonso-Prieto, 2003; Chaná et al., 2013), memoria de trabajo (Lewis et al., 2003), planificación y flexibilidad cognitiva (Cools et al., 2001). Por su parte, Altgassen et al. (2007) han planteado que la alteración en tareas de planificación involucra procesos relacionados con la integración de la información de la memoria de corto y largo plazo. Además, se ha establecido una relación entre una disminución de la dopamina en la proyección nigro-estriatal y el rendimiento ejecutivo en estos sujetos (Marie et al., 1999); de hecho, se ha observado que el rendimiento aumenta con medicación dopaminérgica debido al aumento del flujo en el córtex prefrontal (Cools et al., 2001; Yahr et al., 1969).

Este cuadro tiene repercusiones en diversos aspectos del comportamiento de estos sujetos, dentro de los que se cuenta el lenguaje (Grossman et al., 2000; Lee et al., 2003). En efecto, variadas investigaciones han observado el vínculo entre alteraciones en determinados niveles de la lengua y alteraciones neurológicas y cognitivas (Altmann y Troche, 2011). Entre estas evidencias destaca la capacidad de evocar unidades léxicas y recuperarlas desde el Lexicón, pues se ha evidenciado que este tipo de tareas parecen ser sensibles para la detección del Parkinson, tanto en pacientes con deterioro cognitivo leve como con demencia (Biundo et al., 2014).

El estudio de las funciones cognitivas y lingüísticas en esta población permite contar con mayores descripciones del curso de las alteraciones desde el periodo en que la patología se encuentra con ausencia de sintomatología (pródromo) hasta su disfunción. Asimismo, y tal como se realiza en otras patologías (Comesaña y Vivas, 2015), es relevante ampliar la búsqueda de marcadores cognitivos, con el fin de anticipar el diagnóstico y mejorar el pronóstico en esta población, que epidemiológicamente va en aumento (Liu, Locascio y Corvol, 2017).

\section{La fluidez verbal en el ámbito psicolingüístico}

La prueba de fluidez verbal (FV), como cualquier constructo neuropsicológico, puede ser definida tanto desde los modelos de procesamiento de la información como desde su medición empírica.

De este modo, por un lado, la FV se define como una función ejecutiva que involucra el acceso y evocación a clusters de información lingüística, relacionada con procesos atencionales y memoria de trabajo. Así, se señala que la FV se despliega gracias a la activación de grupos de información mediante estrategias de búsqueda específica, e involucra funciones lingüísticas como la denominación y funciones ejecutivas de planificación, memoria de trabajo e iniciación de conducta (Pulvermüller, 2001; Ruff et al., 1997).

Por otro lado, la FV puede ser entendida como el producto de una tarea de evocación que permite elicitar las funciones ejecutivas, cuya implementación es de gran utilidad dada su simpleza e interpretabilidad de sus resultados (Bolla et al., 1990; Troyer, 2000).

Más allá de esta discusión, como paradigma de evaluación las tareas de FV consisten en solicitar al sujeto que nomine la mayor cantidad de elementos de una categoría léxica de acuerdo a una regla durante un tiempo determinado (ej: palabras que nominen animales que comiencen con el fonema /a/ durante un minuto). Particularmente, la tarea de regla fonológica se identifica como aquella que permite medir las habilidades metafonológicas (Gombert, 1992). A partir de su aplicación se ha observado activación de zonas asociadas al lenguaje, específicamente la zona ventral-anterior del giro frontal inferior (área 45 de Brodmann) en tareas categoriales y la zona dorsal-posterior del giro frontal inferior (área 44 de Brodmann) en tareas fonológicas. Asimismo, cuando las tareas incrementan su dificultad, se ha observado activación de zonas parietales del hemisferio derecho que se asocian a funciones ejecutivas y a procesos 
atencionales (Costafreda et al., 2006; Dräger et al., 2004; Heim, Eickhoff y Amunts, 2008).

Dentro de las aplicaciones de estas tareas se encuentran las investigaciones a lo largo del ciclo vital. Así, por ejemplo, Costafreda et al. (2006) observaron diferencias de rendimiento en la evocación de palabras con fonema excluido por parte de adultos mayores. LópezHiges et al. (2014) compararon el rendimiento de sujetos con deterioro cognitivo leve y controles sanos, a través de una tarea de nominación de animales en 15 y 60 segundos, $\mathrm{y}$ analizaron el tamaño de las agrupaciones subcategoriales y el número de cambios intercategoriales; las conclusiones del estudio mostraron que los sujetos con deterioro solo se diferenciaron de los controles en la producción de una menor cantidad de palabras.

Asimismo, se ha observado rendimiento alterado en pacientes con demencia tipo Alzheimer. En este caso Bryan, Luszcz y Crawford (1997) concluyeron que las alteraciones en la memoria semántica pueden ser una consecuencia de un déficit en el acceso a la información más que en el almacenamiento de la memoria semántica. En esta misma línea, también se ha observado un rendimiento significativamente menor en sujetos con afasia y se han encontrado evidencias que indican que los resultados en FV por 30 segundos permitiría diferenciar sujetos con daño cerebral de otros sin afasia (Kim et al., 2011). Junto a estas evidencias clínicas, García-Laredo et al. (2015) encontraron una relación directa entre el rendimiento en tareas de FV del subtipo semántica categorial con el nivel educacional de los sujetos, lo que indicaría que este factor no debe ser subestimado en estos análisis.

\section{Fluidez verbal y enfermedad de Parkinson}

Los datos sobre la fluidez verbal en los sujetos con EP parecen contradictorios. Así, mientras que Flowers, Robertson y Sheridan (1995) y Azuma et al. (1997) observaron un déficit en la FV de subtipo fonológica, Auria- combe et al. (1993) y Bott et al. (2014) no encontraron diferencias significativas entre sujetos con EP sin demencia y controles sanos en este mismo tipo de tareas. Frente a ello, Henry y Crawford (2004), a partir de un metaanálisis en que se revisaron 68 investigaciones sobre 4644 sujetos, señalan que los sujetos con EP efectivamente presentan un rendimiento inferior, pero que esta diferencia solo se evidencia en tareas de tipo semántica -y no fonológica-, ya que estos sujetos presentan una alteración en la memoria semántica. Ahora bien, este rendimiento deficitario de los sujetos con EP está asociado a la hipofunción del córtex prefrontal, que permite la planificación, el uso de estrategias y el control inhibitorio, funciones requeridas en la evocación de unidades léxicas y en el descarte de otras que no cumplan con los requisitos de la tarea (Baldo y Shimamura, 1998).

Otro aspecto contradictorio se relaciona con la activación cerebral generada a partir de la evocación semántica. Así, Piatt, Fields, Paolo y Tröster (2004) y Ostberg et al. (2007) han asociado este subtipo de FV a lesiones posteriores de la corteza, particularmente en zonas parietales del hemisferio izquierdo. No obstante, Heim, Eickhoff y Amunts (2009) y de Zubicaray y McMahon (2009) establecieron que la evocación de palabras, independiente de su tipo, involucra áreas mediales de la corteza frontal izquierda.

Estas evidencias dispares parecen indicar que los resultados que obtienen los sujetos con EP podrían deberse al subtipo de tarea de FV que se realiza, las cuales generarían diferentes vías de activación, dado el tipo de evocación involucrada. Dicho de otro modo, la patogenia y -por lo tanto- la hipofunción cortical o subcortical, podría ser la causa del rendimiento diferenciado en tareas similares, pero que involucran funciones lingüísticas específicas.

Estos antecedentes llevan a plantear dos posibles hipótesis de rendimiento de los sujetos con EP frente a tareas de FV de diferentes subtipos: por un lado, si los sujetos con 
EP obtienen resultados similares sin importar el nivel lingüístico al que apunte la tarea, implicará alteraciones en la evocación de unidades; por otro lado, si los sujetos con EP obtienen resultados diferenciados por subtipo de tarea, implicará alteraciones específicas en la activación de unidades.

Sin embargo, hasta ahora solo se cuenta con datos sobre subtareas de FV respecto de poblaciones sin patología (Marino y Alderete, 2010). En este marco, el objetivo de este estudio consistió en determinar el rendimiento de dos grupos de adultos mayores, uno compuesto por sujetos con EP y otro por sujetos sin ella, en tareas de FV de varios tipos: fonológica, morfosintáctica, semántica y sus combinaciones, a fin de analizar el compromiso en la activación de unidades específicas.

\section{Método}

\section{Participantes}

Se realizó un estudio transversal con 42 sujetos, agrupados en adulto mayor sano (AMS; $n=23$ ) y adulto mayor diagnosticado con EP sin demencia $(n=19)$. Los sujetos del grupo control tenían una media de edad de 66 años $(D E=6.9)$ y de 11.5 años de escolaridad $(D E=3.1)$; por su parte, los sujetos con EP presentaron una media de edad de 71 años $(D E=8.03)$ y de 13.2 años de escolaridad $(D E=3.9)$.

Los sujetos del grupo AMS fueron reclutados en asociaciones de vecinos y agrupaciones sociales, y los criterios de exclusión fueron presentar patologías neurológicas o psiquiátricas y no estar dispuestos a participar. Para determinar su idoneidad, fueron evaluados mediante el screening cognitivo Mini Mental State Examination (MMSE), que valora la orientación temporo-espacial, atención, memoria, abstracción, lenguaje y visoespacialidad (Quiroga, Albala y Klaasen, 2004), y, siguiendo la norma chilena, se estableció la indemnidad cognitiva sobre los 26 puntos. Los sujetos EP fueron reclutados en la organización Liga Chilena del Parkinson, en Santiago de Chile. Los criterios de inclusión fueron presentar Enfermedad de Parkinson en etapa leve, con MMSE superior a 26 puntos y voluntad de participar en el estudio, y los criterios de exclusión fueron presentar demencia, deterioro cognitivo leve, disartria u otra comorbilidad neuropsiquiátrica. Esta información se obtuvo de evaluaciones cognitivas actualizadas, realizadas por los profesionales de la Liga Chilena del Parkinson.

Este estudio fue aprobado por el Comité de Ética Científica de la Universidad Santo Tomás, Chile, con el código de investigación 55.15. Todos los participantes firmaron un consentimiento informado.

\section{Instrumentos}

Siguiendo el paradigma de Marino y Alderete (2010) de evaluación de la fluidez verbal, cada sujeto realizó un total de 15 subtareas, que se describen en la Tabla 1.

Como se desprende de la discusión teórica planteada más arriba, este paradigma y las subtareas asociadas permiten dar cuenta del procesamiento lingüístico y sus vías de activación. 
Tabla 1. Subtareas de Fluidez Verbal.

\begin{tabular}{|c|c|c|c|}
\hline Tipo de evocación & Condición de evocación & Consigna & Código \\
\hline \multirow{5}{*}{ Fonológica } & \multirow{3}{*}{ Letra designada inicial } & Diga palabras que comiencen con la letra $\mathrm{F}$ & FLD 1 \\
\hline & & Diga palabras que comiencen con la letra $\mathrm{P}$ & FLD 2 \\
\hline & & Diga palabras que comiencen con la letra $\mathrm{A}$ & FLD 3 \\
\hline & \multirow{2}{*}{ Letra excluida de la palabra } & Diga palabras que no contengan $\mathrm{E}$ & FLE 1 \\
\hline & & Diga palabras que no contengan $\mathrm{M}$ & FLE 2 \\
\hline \multirow{2}{*}{$\begin{array}{l}\text { Conciencia } \\
\text { fonológica }\end{array}$} & Número de sílabas (2) & Diga palabras compuestas por 2 sílabas & FCF 1 \\
\hline & Número de fonemas (5) & Diga palabras compuestas por 5 letras* & FCF 2 \\
\hline \multirow{3}{*}{ Morfosintáctica } & Categoría gramatical (verbos) & Diga palabras que sean verbos & M 1 \\
\hline & Categoría gramatical (adjetivos) & Diga palabras que sean adjetivos & M 2 \\
\hline & $\begin{array}{l}\text { Derivación morfológica } \\
\text { (sufijación) }\end{array}$ & Diga palabras que terminen en -ero & M 3 \\
\hline \multirow{3}{*}{ Semántica } & Campo léxico (animales) & Diga todos los nombres de animales que conozca & S 1 \\
\hline & Campo léxico (ciudades) & Diga todos los nombre ciudades de Chile que conozca & S 2 \\
\hline & Relaciones semánticas (sinonimia) & Diga pares de palabras que sean sinónimas & S 3 \\
\hline $\begin{array}{l}\text { Semántica - } \\
\text { fonológica }\end{array}$ & Campo léxico + letra inicial & Diga nombres de mujeres que comiencen con $\mathrm{C}$ & $\mathrm{SF}$ \\
\hline $\begin{array}{l}\text { Morfosintáctico- } \\
\text { fonológico }\end{array}$ & Categoría gramatical + verbo & Diga verbos que no contengan $A$ & SM \\
\hline
\end{tabular}

Nota: Dado que los hablantes están menos familiarizados con las categorías fonológicas (fonemas) que con sus representaciones gráficas (letras), se optó por nombrar estas últimas a fin de que los pacientes pudieran comprender la consigna.

\section{Procedimiento}

Cada una de estas subtareas y el conteo de unidades evocadas se realizaron bajo las siguientes condiciones:

- Cada aplicación se realizó en un contexto libre de distracciones y ruidos.

- Antes de iniciar la evaluación, se explicó la tarea y su duración por ítem. Se entregó siempre la misma instrucción. Asimismo, se ejemplificó con una tarea tipo que no fue contabilizada.

- Todos los sujetos tuvieron 60 segundos para responder. No hubo interrupciones durante la tarea.

- Se consignaron las respuestas cada 15 segundos.

Algunas respuestas fueron descartadas: en las fonológicas no se contabilizaron nombres 
de personas, variaciones de género gramatical, número o afijación; en las semánticas no se contabilizaron, variaciones de género, sinonimia, neologismos ni parafasias fonémicas, para el caso de los animales; no se aceptaron otras divisiones ni regiones geográficas, para el caso de las ciudades; en las morfosintácticas no se contabilizó la derivación morfológica en verbos ni adjetivos; en la fonológica-semántica no se contabilizaron los nombres propios ni los que fueran de origen hispano.

Para el análisis de datos se realizó una descripción de rendimientos $\mathrm{y}$, posteriormente, para establecer la normalidad de distribución de los datos se utilizó Kolmogorov-Smirnov. Luego, para determinar diferencias significativas entre los grupos, se utilizó el test no paramétrico de U de Mann Whitney. Finalmente, se realizó una correlación de Spearman. Todo el análisis estadístico se realizó utilizando el programa IBM SPSS.

\section{Resultados}

La Tabla 2 muestra medias, desviación estándar y coeficiente de variación de los grupos en cada una de las tareas, así como la comparación de medias.

Como muestra la Tabla 2, en cuanto a los totales, en cada una de las pruebas el grupo AMS mostró desempeños promedio más altos que el grupo EP, a excepción de las tareas de letra designada, en las que las medias son similares o levemente superiores. En la desviación estándar y coeficientes de variación se observa que ambos grupos se comportan de manera heterogénea. Se encontraron diferencias significativas en la comparación de medias solo en las pruebas fonológicas de fonema excluido (FVFLE1: $p=.012$ y FVFLE2: $p=.047$ ), en la morfosintáctica de categorías adjetivas (FVM2: $p=.005$ ), en la de relaciones sinonímicas (FVS3: $\mathrm{p}=.028$ ) y en la de campo léxico y fonología (FVSF: $p=.004)$.

Tabla 2. Comparación de medias de rendimiento por grupo y significancia estadística de las diferencias.

\begin{tabular}{ccccccccc}
\hline & \multicolumn{3}{c}{ AMS } & & & EP & & Cohen \\
\hline FLD 1 & 12.87 & 5.25 & $40.7 \%$ & 13.74 & 6.23 & $45.3 \%$ & .486 & .1 \\
\hline FLD 2 & 14.09 & 5.08 & $36.0 \%$ & 15 & 8.03 & $53.5 \%$ & .849 & .1 \\
\hline FLD 3 & 12.35 & 4.68 & $37.8 \%$ & 12.42 & 6.14 & $49.4 \%$ & .949 & 0 \\
\hline FLE 1 & 11.3 & 3.88 & $34.3 \%$ & 8.11 & 3.9 & $48.1 \%$ & $.012 *$ & .3 \\
\hline FLE 2 & 15.13 & 4.31 & $28.4 \%$ & 11.53 & 6.39 & $55.4 \%$ & $.047 *$ & .3 \\
\hline FCF 1 & 12.7 & 6.90 & $54.3 \%$ & 9.84 & 6.18 & $62.8 \%$ & .270 & .2 \\
\hline FCF 2 & 6.43 & 3.75 & $58.3 \%$ & 4.89 & 4.89 & $100.0 \%$ & .099 & .2 \\
\hline M 1 & 12.09 & 7.33 & $60.6 \%$ & 11.26 & 5.36 & $47.6 \%$ & .939 & .1 \\
\hline M 2 & 13.22 & 5.80 & $43.8 \%$ & 7.95 & 5.72 & $71.9 \%$ & $.005 *$ & .4 \\
\hline M 3 & 7.30 & 3.60 & $49.3 \%$ & 7.37 & 3.67 & $49.8 \%$ & .819 & 0 \\
\hline S 1 & 20.13 & 6.09 & $30.2 \%$ & 16.95 & 4.88 & $28.8 \%$ & .128 & .2 \\
\hline S 2 & 19.22 & 6.05 & $31.4 \%$ & 18.11 & 9.01 & $49.8 \%$ & .486 & .1 \\
\hline S 3 & 5.52 & 3.00 & $54.3 \%$ & 3.53 & 2.06 & $58.4 \%$ & $.028 *$ & .3 \\
\hline SF & 8.61 & 4.11 & $47.7 \%$ & 5.37 & 2.56 & $47.7 \%$ & $.004 *$ & .4 \\
\hline SM & 5.09 & 3.65 & $71.7 \%$ & 3.32 & 2.65 & $79.8 \%$ & .257 & .2 \\
\hline
\end{tabular}

* Se rechaza la hipótesis nula. 


\section{Discusión}

El objetivo de este estudio fue determinar el rendimiento de sujetos con EP y controles sanos equiparables, en tareas de FV fonológica, morfosintáctica, semántica y sus combinaciones. En términos generales las medias del grupo AMS fueron levemente superiores a las del grupo EP, a excepción de las tres tareas fonológicas en las que estos últimos obtuvieron medias superiores. Solamente se encontraron diferencias significativas en algunas de las tareas de evocación: fonológica de letra excluida (fonema excluido), morfosintáctica de adjetivos (categoría gramatical), semántica de pares de sinónimos (relaciones semánticas) y de combinación semántica-fonológica (campo léxico y fonema inicial). Frente a ello, las tareas FVFLD1, FVFLD2, FVFLD3, FVFCF1, FVFCF2, FVM1, FVM3, FVS1, FVS2 y FVSM no permitieron observar un comportamiento diferencial de los sujetos con EP.

En las tareas de conciencia fonológica, no se encontraron diferencias en las de fonema inicial /f/ /p/ /a/, en consonancia con lo señalado por Auriacombe (2004), Bott (2014) y Henry y Crawford (2004). Por el contrario, sí se encontraron diferencias significativas en las pruebas de fonema excluido $/ \mathrm{e} / \mathrm{y} / \mathrm{m} / \mathrm{La}$ diferencias entre ambos tipos de tarea radica en que estas últimas (FVFLE1 y FVFLE2) son más demandantes en términos psicolingüísticos, ya que, una vez evocada la unidad léxica, el sujeto debe volver sobre ella, segmentarla en unidades fonológicas, analizarla y aceptarla o rechazarla, según si cumple o no la regla fonológica que exige la tarea. En cambio, en el caso de las tareas de fonema inicial designado, el sujeto debe evocar bajo una regla y luego inhibir las unidades no correspondientes. Esta diferencia en el costo ejecutivo puede explicar las diferencias del comportamiento de los sujetos con EP frente a uno y otro tipo de tarea fonológica.

En las tareas de conciencia fonológica no se observaron diferencias significativas en las de segmentación silábica (FVFCF1) ni en las de segmentación vocálica (FVFCF2), aunque en las medias son superiores los sujetos del grupo AMS.

En las tareas morfosintácticas solo se observó una diferencia significativa en la de categorías adjetivas (FVM2), mientras que en las tareas de verbos (FVM1) y sufijación (FVM3) no se observaron diferencias. Una posible explicación podría estar en la disponibilidad y saliencia de las categorías gramaticales en la lengua española. De hecho, los adjetivos son categorías menos numerosas que otras, como los verbos por ejemplo. No obstante, no se cuenta con datos psicolingüísticos que puedan avalar esta afirmación.

En cuanto a la tarea morfosintáctica de sufijación (FVM3), los sujetos con EP tienen un desempeño levemente superior a los sujetos AMS y no se presentaron diferencias significativas. Ahora bien, es necesario destacar que esta tarea resultó altamente exigente para ambos grupos, pues las medias no superan las 8 palabras por minuto, a pesar de que desde el punto de vista psicolingüístico se podría especular que la sufijación es una tarea altamente demandante y que los sujetos EP deberían tener un rendimiento inferior. Estos datos indican que sería necesario ahondar en este aspecto en futuras investigaciones.

En cuanto a las tareas semánticas, no mostraron diferencias las de campo léxico (FVS1 y FVS2), mientras que en la de relaciones semánticas (FVS3) se encontró diferencia significativa. En términos lingüísticos, el campo léxico y las relaciones de sinonimia son distintos tipos de relaciones semánticas. En el primer caso, las unidades que componen el conjunto comparten, al menos, un sema que rige la configuración del conjunto de unidades; por ejemplo, en el caso de las unidades [pantalón, camisa, zapatos, corbata] el rasgo compartido de estas unidades léxicas es que sean prendas de vestir (Coseriu, 1977). En el caso de las relaciones de sinonimia, las unidades léxicas denotan las mismas entidades, como lo hacen, por ejemplo, los pares 
de palabras [perro/can, pelo/cabello, blanco/ albo] (Cruse, 1986). Ahora bien, no existe ningún motivo para afirmar que las relaciones de campo léxico sean más complejas o sencillas que las de relaciones de sentido de sinonimia; sin embargo, si volvemos al tipo de tarea nos encontramos que en FVS1 y FVS2 el sema compartido fue explicitado a los sujetos (ciudades y animales, respectivamente), mientras que en el caso de FVS3 los sujetos no contaron previamente con el rasgo semántico que debían utilizar para evocar los pares de palabras. Dicho de otro modo, la tarea FVS3 es más exigente que las anteriores, dado que el sujeto debe evocar un par de unidades léxicas y decidir el rasgo que las une, es decir, además se debe realizar una búsqueda, inhibición y selección de la relación semántica que se debe establecer.

En las tareas combinadas sería esperable encontrar diferencias significativas en ambas subtareas, dada la demanda ejecutiva de estas. No obstante, en FVSM no ocurrió. Una explicación posible para este hecho está dada por el alto grado de dificultad que representó para el grupo AMS, que no superó las 5 palabras por minuto.

Por último, se realizó para todas las tareas un análisis de cantidad de unidades evocadas en intervalos de 15 segundos y no se observaron patrones de interés. Por otra parte, la desviación estándar y los coeficientes de variación presentaron un comportamiento heterogéneo en cada una de las tareas. Esto indica que sería necesario replicar este estudio con poblaciones mayores, para observar la normalidad del comportamiento de las poblaciones en estudio, pues esta heterogeneidad influye en los resultados inferenciales hasta aquí descriptos.

Como se ha visto hasta acá, en general, las diferencias significativas entre los grupos pueden explicarse por la complejidad tanto lingüística como cognitiva de las tareas y por alteraciones en la evocación de unidades. De hecho, cada vez que los sujetos con EP se han enfrentado a tareas que exigen utilizar estrategias de evocación, seleccionar unidades léxicas y recuperarlas desde el Lexicón, y en forma paralela han debido inhibir otros candidatos léxicos, se han observado diferencias significativas. En este sentido, las diferencias observadas entre EP y AMS pueden ser explicadas debido a la complejidad de la tarea y al déficit ejecutivo que se asocia a procesos dopaminérgicos en el córtex orbital que presentan déficit los sujetos con EP. No obstante, no todas las tareas han permitido dar cuenta de este hecho.

\section{Referencias bibliográficas}

Alonso Prieto, E., Esteban, E., Trujillo Matienzo, C., Lara Fernández, G., Rousso Viota, T. y Cordero Eiriz, A. (2003). Specific alterations in attention in the early stages of Parkinson's disease. Revista de Neurología, 36, 1015-1018.

Altmann, L. y Troche, M. (2011). High-level language production in Parkinson's disease: a review. Parkinson's Disease, 2011, 238956. doi: 10.4061/2011/238956

Altgassen, M., Phillips, L., Kopp, U. y Kliegel, M. (2007). Role of working memory components in planning performance of individuals with Parkinson's disease. Neuropsychologia, 45, 2393-2397.

Auriacombe, S., Grossman, M., Carvell, S., Gollomp, S., Stern, M. y Hurtig, H. (1993). Verbal fluency deficits in Parkinson's disease. Neuropsychology, 7, 182-192.

Azuma, T., Bayles, K., Cruz, R., Tomoeda, C., Wood, J., Mcgeagh, A. y Montgomery, E.B. Jr. (1997). Comparing the difficulty of letter, semantic, and name fluency tasks for normal elderly and patients with Parkinson's disease. Neuropsychology, 11, 488-497.

Baldo, J. y Shimamura, A. (1998). Letter and category fluency in patients with frontal lobe lesions. Neuropsychology, 12, 259-267.

Biundo, R., Weis, L., Facchini, S., Formento-Dojot, P., Vallelunga, A., Pilleri, M. y Antonini, A. (2014). Cognitive profiling of Parkinson disease patients with mild cognitive 
impairment and dementia. Parkinsonism and Related Disorders, 20,394-399. doi: 10.1016/j. parkreldis.2014.01.009

Bolla, K., Lingren, L., Bonaccorsy, C. y Bleecker, M. (1990). Predictors of verbal fluency (FAS) in the healthy elderly. Journal of Clinical Psychology, 46, 623-628.

Bott, N., Johnson, E., Schuff, N., Galifianakis, N., Subas, T., Pollock, J., Pressman, P., Kramer, J. y Possin, K. (2014). Sensitive measures of executive dysfunction in non-demented Parkinson's disease. Parkinsonism and Related Disorders, 20, 1430-1433. doi: 10.1016/j. parkreldis.2014.10.007

Bryan, J., Luszcz, M. y Crawford, J. (1997). Verbal knowledge and speed of information processing as mediation of age differences in verbal fluency performance among older adults. Psychology and Aging, 12, 473-478.

Calne, D.B., Snow, B.J. y Lee, C. (1992). Criteria for diagnosing Parkinson's disease. Annals of Neurology, 32, 125-127.

Chaná, P.C., González, H.A., Gutiérrez, A. y Cáceres, C. (2013). Parámetros cinemáticos en la prueba de Tapping en pacientes con enfermedad de Parkinson. Revista Chilena de Neuropsiquiatría, 51, 95-101. doi: 10.4067/ S0717-92272013000200002

Comesaña, A. y Vivas, J. (2015). Evolución de la categorización semántica en adultos mayores con diagnóstico de DCL-A y DTA y sin patología neurológica. Interdisciplinaria, Revista de Psicología y Ciencias Afines, 32, 7-29. doi: 10.16888/interd.2015.32.1.1

Cools, R., Barker, R., Sahakian, B. y Robbins, T. (2001). Mechanisms of cognitive set flexibility in Parkinson's disease. Brain, 124, 2503-252.

Costafreda, S., Fu, C., Lee, L., Everitt, B., Brammer, M. y David, A. (2006). A systematic review and quantitative appraisal of fMRI studies of verbal fluency: role of the left inferior frontal gyrus. Human Brain Mapping, 27, 799-810.

Cruse, D.A.(1986). Lexical semantics. Cambridge: University Press.

Coseriu, E. (1977). Principios de semántica estructural. Madrid: Gredos.
De Zubicaray, G. y Mcmahon, K. (2009). Auditory context effects in picture naming investigated with event-related fMRI. Cognitive, Affective and Behavioral Neuroscience, 9, 260-269. doi: 10.3758/CABN.9.3.260

Dräger, B., Jansen, A., Bruchmann, S., Förster, A., Pleger, B., Zwitserlood, P. y Knecht, S. (2004). How does the brain accommodate to increased task difficulty in word finding? A functional MRI study. Neuroimage, 23, 1152-1160.

Dubois, B. y Pillon, B. (1996). Cognitive deficit in Parkinson's disease. Journal of Neurology, 244, 2-8.

Flowers, K., Robertson, C. y Sheridan, M. (1995). Some characteristics of word fluency in Parkinson's disease. Journal of Neurolinguistics, 9, 33-46.

García-Laredo, E., Maestú, F., Castellanos, M., Molina, J. y Peréz-Moreno, E. (2015). The relationship between educational years and phonemic verbal fluency (PVF) and semantic verbal fluency (SVF) tasks in Spanish patients diagnosed with schizophrenia, bipolar disorder, and psychotic bipolar disorder. Medicine, 94, e1596. doi: 10.1097/MD.0000000000001596.

Gombert, J.E. (1992). Metalinguistic development. New York, NY: Harvester Wheatsheaf.

Grossman, M., Kalmanson, J., Bernhardt, N., Morris, J., Stern, M.B., y Hurtig, H.I. (2000). Cognitive resource limitations during sentence comprehension in Parkinson's disease. Brain and Language, 73, 1-16.

Heim S., Eickhoff, S. y Amunts, K. (2008). Specialisation in Broca's region for semantic, phonological, and syntactic fluency? Neuroimage, 40, 1362-1368. doi: 10.1016/j.neuroimage.2008.01.009.

Heim S., Eickhoff, S. y Amunts, K. (2009). Different roles of cytoarchitectonic BA44 and BA45 in phonological and semantic verbal fluency as revealed by dynamic causal modelling. Neuroimage, 48, 616-662. doi: 10.1016/j. neuroimage.2009.06.044.

Henry, J. y Crawford, J. (2004). Verbal fluency deficits in Parkinson's disease: a meta-analysis. Journal of the International Neuropsychological Society, 10, 608-622. 
Kim, H., Kim, J., Kim, D. y Heo, J. (2011). Differentiating between aphasic and nonaphasic stroke patients using semantic verbal fluency measures with administration time of 30 seconds. European Neurology, 65, 113-117. doi: 10.1159/000324036

Lee, C., Grossman, M., Morris, J., Stern, M.B. y Hurtig, H.I. (2003). Attentional resource and processing speed limitations during sentence processing in Parkinson's disease. Brain and Language, 85, 347-356.

Lewis, S., Cools, R., Robbins, T., Dove, A., Barker, R. y A. Owen. (2003). Using executive heterogeneity to explore the nature of working memory deficits in Parkinson's disease. Neuropsychologia, 41, 645-654.

Lippa, C.F., Duda, J.E., Grossman, M., ... y Wszolek, Z.K. (2007). DLB and PDD boundary issues. Diagnosis, treatment, molecular pathology, and biomarkers. Neurology, 13, 812-819. doi: 10.1212/01. wnl.0000256715.13907.d3

Liu, G., Locascio, J.J. y Corvol, J.C., PDBP. (2017) Prediction of cognition in Parkinson's disease with a clinical-genetic score: a longitudinal analysis of nine cohorts. Lancet Neurology, 16, 620-629. doi: 10.1016/S14744422(17)30122-9.

López-Higes, R., Prados, J., Del Río, D., Galindo-fuentes, M., Reinoso, A. y Lozano-Ibánez, M. (2014). Semantic verbal fluency of animals in amnesia-type mild cognitive impairment. Revista de Neurología, 58, 493-499. doi: 10.33588/rn.5811.2014048

Marie, R., Barre, L., Dupuy, B. y Viader. F. (1999). Relationships between striatal dopamine denervation and frontal executive tests in Parkinson's disease. Neuroscience Letters, 260, 77-80.

Marino, J. y Alderete, A. (2010). Valores normativos de pruebas de fluidez verbal categoriales, fonológicas, gramaticales y combinadas y análisis comparativo de la capacidad de Inici- ación. Revista Neuropsicología, Neuropsiquiatría y Neurociencias, 10, 79-93.

Ostberg, P., Crinelli, R., Danielsson, R., Wahlund, L., Bogdanovic, N., y Fernaeus, S. (2007). A temporal lobe factor in verb fluency. Cortex, 43, 607-615.

Perfetti, B., Varanese, S., Mercuri, P., Mancino, F., Saggino, A. y Onofrj, M. (2010). Behavioural assessment of dysexecutive syndrome in Parkinson's disease. Parkinsonism and Related Disorders, 16, 46-50. doi: 10.1016/j. parkreldis.2009.07.011.

Petrova, M., Raycheva, M., Zhelev, Y. y Traykov, L. (2010). Executive functions deficit in Parkinson's disease with amnestic mild cognitive impairment. American Journal of Alzheimer's Disease and Other Dementias, 25, 455-460. doi: 10.1177/1533317510370956.

Piatt, A., Fields, J., Paolo, A. y Tröster, A. (2004). Action verbal fluency normative data for the elderly. Brain and Language, 89, 580-583.

Pulvermüller, F. (2001). Brain reflections of words and their meaning. Trends in Cognitive Sciences, 5, 517-524.

Quiroga, P., Albala, C. y Klaasen, G. (2004). Validación de un test de tamizaje para el diagnóstico de demencia asociada a edad en Chile. Revista Médica de Chile, 132(4), 467-478.

Ruff, R., Ligth, R., Parker, S. y Levin, H. (1997). The psychological construct of word fluency. Brain and Language, 57, 349-405.

Taylor, A., Saint-Cyr, J. y Lang, A. (1986). Frontal lobe dysfunction in Parkinson's disease. The cortical focus of neostriatal outflow. Brain, 109, 845-883.

Troyer, A. (2000). Normative data for clustering and switching on verbal fluency tasks. Journal of Clinical and Experimental Neuropsycholy, 22, 370-378.

Yahr, M.D., Duvoisin, R.C., Schear, M.J., Barrett, R.E. y Hoehn, M.M. (1969). Treatment of parkinsonism with levodopa. Archives of Neurology, 21, 343-354.

Recibido: 8 de enero de 2018 Aceptado: 30 de septiembre de 2019 
